\title{
C20orf20 (MRG-binding protein) as a potential therapeutic target for colorectal cancer
}

\author{
K Yamaguchi ${ }^{1,4}$, M Sakai ${ }^{2,4}$, T Shimokawa ${ }^{2}$, Y Yamada $^{3}$, Y Nakamura ${ }^{2}$ and Y Furukawa*, I \\ 'Division of Clinical Genome Research, Advanced Clinical Research Center, Institute of Medical Science, The University of Tokyo, 4-6-I Shirokanedai, \\ Minato-ku, Tokyo 108-8639, Japan; ${ }^{2}$ Laboratory of Molecular Medicine, Human Genome Center, Institute of Medical Science, The University of Tokyo, \\ 4-6-I Shirokanedai, Minato-ku, Tokyo 108-8639, Japan; ${ }^{3}$ Department of Anesthesiology, School of Medicine, The University of Tokyo, 7-3-I Hongo, \\ Bunkyo-ku, Tokyo I/ 3-0033, Japan
}

BACKGROUND: Colorectal cancer is one of the most common causes of cancer death worldwide. Using cDNA microarray containing 23040 genes, we earlier investigated gene-expression profiles in II colorectal cancers for the purpose of better understanding of colorectal carcinogenesis as well as development of novel diagnostic and therapeutic strategies. MRG-binding protein (MRGBP) or C20orf20, encoding a subunit of TRRAP/TIP60-containing histone acetyltransferase complex, was up-regulated in the majority of colorectal tumours.

METHODS AND RESULTS: The elevated expression of MRGBP was observed in colorectal cancer tissues by quantitative PCR as well as immunohistochemical analyses. MRGBP marginally expressed in normal vital organs. Notably, suppressed MRGBP expression by MRGBP short hairpin RNA inhibited proliferation of colorectal cancer cells. Yeast two-hybrid screening and subsequent immunoprecipitation analysis identified bromodomain containing 8 (BRD8) as an MRGBP-interacting protein. As RNA interference against BRD8 also suppressed proliferation of colorectal cancer cells, BRD8 may be an important down-stream target of MRGBP. CONCLUSION: These results suggest that MRGBP has an important function in proliferation of cancer cells through the regulation of BRD8 and that MRGBP should be a novel therapeutic target for colorectal cancer.

British Journal of Cancer (2010) I 02, 325-331. doi:I0.I038/sj.bjc.6605500 www.bjcancer.com

Published online 5 January 2010

(C) 2010 Cancer Research UK

Keywords: C20orf20 (MRGBP); BRD8; colorectal cancer; cell proliferation

Colorectal cancer is the second leading cause of cancer death in the United States, and its incidence rates are increasing in Japan. It is estimated that there are almost 500000 colorectal cancer-related deaths every year in the world (Parkin, 2001). Although recent medical advances have improved the prognosis of patients with the disease, complete cure of patients with advanced tumour is far from satisfactory. In chemotherapies for advanced colorectal cancer, oxaliplatin/fluorouracil/leucovorin (FOLFOX) is an effective and well-tolerated regimen. Combination of targeted biological agents such as anti-epidermal growth factor receptor (EGFR) with FOLFOX have been reported to enhance the efficacy against EGFRexpressing metastatic colorectal cancer (Giantonio, 2006; Tabernero et al, 2007). This indicates that the use of rationally selected therapeutic agents will improve the treatment for advanced diseases and results in increase of cure rate and/or prolonged survival. Regarding colorectal cancer, combination chemotherapy with Bevacizumab, an inhibitor of VEGF receptor, was approved in the United States and was shown to be effective for $45 \%$ of patients with colorectal cancer and increased their 1-year survival rate from $63.4 \%$ to $74.3 \%$ (Hurwitz et al, 2004). However, many patients are

\footnotetext{
*Correspondence: Dr Y Furukawa; E-mail: furukawa@ims.u-tokyo.ac.jp

${ }^{4}$ These two authors contributed equally to this work

Revised 12 November 2009; accepted 25 November 2009; published online 5 January 2010
}

still suffering and dying from the disease, and development of additional molecular-targeted anti-cancer drugs is a matter of pressing concern for public health.

These drugs target molecules that are expressed abundantly or exclusively in cancer cells and functioning as an indispensable factor for the growth or survival of cancer cells. For instance, Imatinib (STI571) inhibits several protein kinases such as bcr-abl fusion protein in chronic myelogenous leukaemia, and c-kit in gastrointestinal stromal tumours (O'Dwyer and Druker, 2000). Gefetinib targets the ATP cleft within the EGFR (Wakeling, 2002; Fukuoka et al, 2003; Gridelli et al, 2003; Kris et al, 2003). Trastuzumab is a monoclonal antibody to the HER2/neu receptor, which is overexpressed in $\sim 30 \%$ of breast cancers (Molina et al, 2001). These drugs strikingly suppressed the growth of tumour cells and showed minimum cytotoxic effect in normal cells. Therefore, for the development of molecular-targeted anti-cancer drugs pinpointing cancer cells, identification of molecules that are expressed abundantly in cancer cells and clarification of their function are essential.

Molecular studies have clarified that multiple-step process has an important function in colorectal carcinogenesis, which involves activation of oncogenes such as $K$-ras, and inactivation of tumour suppressor genes such as $p 53$ and $A P C$. In addition to these genetic changes, alteration of gene expression is involved in the carcinogenesis. Epigenetic alterations including aberrant DNA 
methylation and/or histone modification have been recently shown to participate in some of the deregulated gene expression. To unveil the molecular mechanisms of colorectal cancer and discover target molecules for the development of novel anti-cancer drugs, we analysed global gene-expression profiles of colorectal tumours by cDNA microarray analysis representing 23040 genes (Lin et al, 2002). These efforts have identified a number of genes, which are frequently either up-regulated or down-regulated in the tumours compared with the corresponding non-cancerous tissues. Among the list of genes up-regulated in the tumours, we found a gene termed as MRG-binding protein (MRGBP), with an approved symbol of chromosome 20 open reading frame 20 (C20orf20), which was identified as a component of TRRAP/TIP60 histone acetyltransferase complex and shown to bind directly to MRG15 and MRGX proteins (Cai et al, 2003). In this report, we show, for the first time, that MRGBP expression was frequently elevated in colorectal cancer, and that it has an important function in the growth of cancer cells. These findings should contribute to a better understanding of colorectal tumourigenesis, and may serve as a starting point for the development of novel strategies for prevention and treatment of colorectal cancer.

\section{MATERIALS AND METHODS}

\section{Cell lines and tissue specimens}

A human embryonic kidney cell line, HEK293, a monkey kidney cell line, COS7, and human colon cancer cell lines, SW480 and HCT116, were obtained from the American Type Culture Collection (Manassas, VA, USA). All cells were grown in monolayers in appropriate media as follows: Dulbecco's modified Eagle's medium for HEK293 and COS7, McCoy's 5A medium for HCT116, and Leibovitz's L-15 for SW480. All media were supplemented with $10 \%$ fetal bovine serum and $1 \%$ antibiotic/ antimycotic solution (Sigma, St Louis, MO, USA). All colorectal cancer tissues and corresponding non-cancerous tissues were obtained with informed consent from surgical specimens of patients who underwent surgery.

\section{Isolation of RNA and quantitative PCR}

Total RNA was extracted with RNeasy kit (Qiagen, Valencia, CA, USA) according to the manufacturers' protocols. One microgram of total RNA was reversely transcribed for single-stranded cDNA using oligo $(\mathrm{dT})_{12-18}$ primer (GE Healthcare, Buckinghamshire, UK) with Superscript II reverse transcriptase (Invitrogen, Carlsbad, CA, USA). Quantitative PCR was carried out using the LightCycler 480 System (Roche Diagnostics, Indianapolis, IN, USA). The probes and primers for $M R G B P$ and hypoxanthine phosphoribosyltransferase1 (HPRT1) are as follows - MRGBP: forward, 5'-GGAGGAGACAGTGGTGTGG-3', reverse, 5'-CATGTG GAAGTGTCGGTTCA-3', and probe, Universal ProbeLibrary \#39 (Roche Diagnostics); HPRT1: forward, 5'-TGACCTTGATTTATTT TGCATACC- $3^{\prime}$, reverse, 5'-CGAGCAAGACGTTCAGTCCT-3', and probe, Universal ProbeLibrary \#73 (Roche Diagnostics).

\section{Northern blot analysis}

HEK293 cells transfected with pCAGGS-HA-bromodomain containing 8 (BRD8) and/or pcDNA-Myc/His-MRGBP were harvested at the indicated time points after transfection. After purification of RNA, $1 \mu \mathrm{g}$ of poly(A) RNA was separated on a $1 \%$ agarose gel containing formaldehyde and transferred to a nylon membrane. The blot was hybridised with ${ }^{32} \mathrm{P}$-labeled PCR product of $M R G B P$ or $\beta$-actin cDNA. Human multiple-tissue northern blots were obtained from BD Biosciences (Palo Alto, CA, USA), and analysed according to the instructions of the manufacturer.
The blots were autoradiographed with intensifying screens at $-80^{\circ} \mathrm{C}$ for 5 days.

\section{Knockdown of endogenous MRGBP expression by RNA interference}

Plasmids expressing short hairpin RNA (shRNA) to MRGBP (psiH1BX-MRGBPs), BRD8 (psiH1BX-BRD8), or EGFP (psiH1BXEGFP) were prepared as described earlier (Shimokawa et al, 2003). Briefly, psiH1BX-MRGBPs and psiH1BX-BRD8 were constructed by the cloning of double-stranded oligonucleotides into psiH1BX vector and the target sequences of synthetic oligonucleotides for $M R G B P$ shRNAs were as follows: $5^{\prime}$-GAGAAUUUGUAGCGGUUA U-3' for shMRGBP\#1, 5'-GUGACAUGGAUUAGCGCUA-3' for shMRGBP\#2, $5^{\prime}$-ACAAAGUCCUGACCGCAAA-3' for shMRGBP\#3, $5^{\prime}$-GGGAGAAGUGGUGGAAACU-3' for shBRD8. To evaluate the knockdown effect on MRGBP and BRD8, SW480 and HCT116 cells were transfected with these shRNA constructs using Nucleofector kit (Amaxa, Gaithersburg, MD, USA), and western blotting was performed. For cell proliferation assay, psiH1BX-MRGBPs, psiH1BX-BRD8, or psiH1BX-EGFP were transfected into SW480 and HCT116 cells using FuGENE6 (Roche Diagnostics) according to the manufacturer's protocol. Transfectants were selected in appropriate concentration of Geneticin (SW480: $1.25 \mathrm{mg} \mathrm{ml}^{-1}$, HCT116: $0.7 \mathrm{mg} \mathrm{ml}^{-1}$ for 7-9 days), and the viable cells were measured by WST-8 assay (Dojindo, Kumamoto, Japan). Control (ON-TARGETplus Non-Targeting pool, Dharmacon, Lafayette, CO, USA) and a mixture of four MRGBP-specific On-Targetplus siRNA oligos (5'-GAGAAUUUGUAGCGGUUAU-3', $5^{\prime}$-GUGACAUGGAU UAGCGCUA- $3^{\prime}, 5^{\prime}$-ACAAAGUCCUGACCGCAAA- $3^{\prime}$, and $5^{\prime}$-CAGG GAAAACCUCGGAUUA-3 ${ }^{\prime}$ ) were also used for the functional analysis.

\section{Flow cytometry}

Cultured colorectal cancer cells were transfected with control or $M R B G P$ siRNA (Dharmacon) for $48 \mathrm{~h}$. For analysis of cellular DNA content, transfected cells were collected and fixed with $70 \%$ ethanol, and then kept at $-20^{\circ} \mathrm{C}$ before use. Cells were incubated with $2 \mathrm{mg} \mathrm{ml}^{-1}$ RNase A at $37^{\circ} \mathrm{C}$ for $30 \mathrm{~min}$ and stained with propidium iodide (PI) at room temperature for $30 \mathrm{~min}$. Assessment of apoptosis by annexin $\mathrm{V}$ and PI double staining was performed using Annexin V-FITC Apoptosis Detection kit (Medical \& Biological Laboratories, Nagoya, Japan). Cellular DNA synthesis was evaluated by incorporation of 5-ethynyl-2'deoxyuridine (EdU) using Click-iT EdU Flow Cytometry Assay kit (Invitrogen). Briefly, transfected cells were cultured in media containing $10 \mu \mathrm{M}$ EdU for $30 \mathrm{~min}$. The incorporated EdU and total DNA were stained with Alexa448-conjugated azide and 7-aminoactinomycin D (7-AAD), respectively. Subsequently, the cell suspensions were analysed on an FACSCalibur (Becton Dickinson, Franklin Lakes, NJ, USA) using FlowJo software (Tree Star, Ashland, OR, USA).

\section{Construction of plasmids expressing MRGBP and BRD8}

The entire coding region of $M R G B P$ and $B R D 8$ were amplified by RT - PCR using gene-specific primer sets. The primer sequences used for the amplification were $5^{\prime}$-TGTGAATTCGCCATGGGAGA GGC-3' (forward) and $5^{\prime}$-TAACTCGAGCGTGCGGCGCCGCTT-3' (reverse) for $M R G B P$, and $5^{\prime}$-ATAGAATTCTCTTCTGTCATGAGA AGTGG- $3^{\prime}$ (forward) and $5^{\prime}$-ATACTCGAGTCACTTTTTCATCTT $\mathrm{C}-3^{\prime}$ (reverse) for BRD8. The cDNA products of $M R G B P$ and $B R D 8$ were cloned into an appropriate cloning site of pcDNA3.1-Myc/His (Invitrogen) or pCAGGS-HA vector, respectively. DNA sequences of all constructs were confirmed by DNA sequencing (ABI3730, Applied Biosystems, Foster City, CA, USA). 
Immunohistochemical staining using polyclonal antibody against MRGBP

We prepared histidine-tagged human MRGBP protein in bacteria, and raised rabbit antibodies specific to MRGBP by immunising rabbits with the MRGBP protein. Purification of antibodies was carried out with standard protocols using affinity columns (Affi-Gel 15, Bio-Rad, Hercules, CA, USA). Specificity of the antibodies was examined by immunoblot analysis using whole extracts from cells expressing Myc-tagged MRGBP (data not shown). Immunohistochemical staining was performed using antiMRGBP polyclonal antibody. Paraffin-embedded tissue sections were subjected to the SAB-PO peroxidase immunostaining system according to the instructions of the manufacturer (Nichirei, Tokyo, Japan).

\section{Immunoprecipitation and western blot analysis}

COS7 and HEK293 cells were transfected with pcDNA-Myc/HisMRGBP, pCAGGS-HA-BRD8, or the combination using FuGENE6. For immunoprecipitation, the cells were lysed in $0.5 \%$ Nonidet P-40 buffer (10 mM Tris $-\mathrm{HCl}$ pH 7.5, $150 \mathrm{~mm} \mathrm{NaCl})$ supplemented with a Protease Inhibitor Cocktail Set III (Calbiochem, San Diego, CA, USA). The whole-cell extract was incubated with anti-Myc (Santa Cruz Biotechnology, Santa Cruz, CA, USA) or anti-HA antibody (Roche Diagnostics), followed by Protein G-Sepharose beads (Invitrogen) at $4^{\circ} \mathrm{C}$. Proteins were separated by SDS-PAGE and immunoblot analysis was performed. Horseradish peroxidase-conjugated goat anti-mouse IgG (GE Healthcare) and goat anti-rat IgG (Santa Cruz Biotechnology) served as the secondary antibody for the ECL Detection System (GE Healthcare). To examine the endogenous interaction of MRGBP and BRD8, nuclear extract from SW480 cells was incubated with anti-MRGBP or anti-p120 (BRD8) antibody (Abcam, Cambridge, UK), followed by Protein G-Sepharose beads overnight at $4^{\circ} \mathrm{C}$. After washing, these immunoprecipitants were applied for SDS-PAGE. Normal rabbit IgG (Santa Cruz Biotechnology) was used as negative control.

\section{Immunocytochemical staining}

COS7 cells were transfected with pcDNA-Myc/His-MRGBP, pCAGGS-HA-BRD8, or the combination of the two. Twenty-four hours after transfection, the cells fixed with $4 \%$ paraformaldehyde were rendered permeable with PBS containing $0.1 \%$ Triton X-100. Subsequently, the cells were covered with $3 \%$ BSA in PBS to block non-specific hybridisation, and incubated with anti-Myc or antiHA antibody. The reaction was visualised after incubation with Alexa Fluor 488 anti-mouse or Alexa Fluor 594 anti-rat secondary antibody (Invitrogen). Nuclei were counterstained with $4^{\prime}, 6^{\prime}$ diamidine-2'-phenylindole dihydrochloride.

\section{RESULTS}

\section{Expression of MRGBP is frequently elevated in colorectal tumours}

We have earlier compared expression profiles of colorectal cancers with the corresponding non-cancerous colon tissues using cDNA microarray and identified a number of up-regulated genes in the cancer cells (Lin et al, 2002). In this study, we investigated a gene termed as MRGBP (formally C20orf20), because its expression was elevated in 9 out of 11 tumours in our microarray data. Reportedly, MRGBP is a subunit of a transcriptional complex of TRRAP/TIP60. Subsequent quantitative PCR confirmed its elevated expression in 10 out of the additional 15 colorectal tumours compared with their matched non-cancerous mucosa (Figure 1A). Western blot analysis also showed enhanced MRGBP expression in 10 out of the additional 14 tumours examined (Figure 1B). Multiple tissue northern blot analysis using $M R G B P$ cDNA as a probe detected a $1.6 \mathrm{~kb}$ transcript that was readily detectable in the skeletal muscle, testis, and thyroid, whereas it showed a relatively low level of expression in important normal organs such as heart, brain, lung, liver, and kidney (Figure 1C).

To further evaluate its expression levels, we performed immunohistochemical staining using 27 colorectal cancer tissues. As a result, we observed accumulated MRGBP mainly in the nucleus of cancer cells in 20 out of the 27 tumours. However, noncancerous epithelial cells in the adjacent mucosa of the tumours did not show accumulation of MRGBP (Figure 1D).

\section{MRGBP confers growth-promoting effect to cancer cells}

To investigate a possible function of elevated MRGBP expression in the proliferation of cancer cells, we prepared plasmids that express MRGBP- and EGFP-specific shRNAs with neomycin resistant gene (psiH1BX-MRGBP1, -MRGBP2, -MRGBP3, and -EGFP). Transfection of SW480 or HCT116 cells with all psiH1BXMRGBPs significantly reduced the MRGBP expression in the cells, whereas that with control plasmid (psiH1BX-EGFP) did not affect MRGBP expression (data not shown). Cells transfected with psiH1BX-MRGBPs or psiH1BX-EGFP were cultured in media containing appropriate concentration of geneticin, and the number of viable cells was examined at day 7 or 9 after the transfection. As a result, psiH1BX-MRGBPs significantly reduced the number of viable cells compared with control plasmid (Figure $2 \mathrm{~A}$ and $\mathrm{B}$ ). To disclose the mechanism(s) underlying the decrease of viable cells by MRGBP knockdown, we investigated induction of apoptosis, cell cycle progression, and DNA synthesis in cancer cells treated with $M R G B P$ siRNA. Knockdown of MRGBP did not influence significantly on population of apoptotic cells (data not shown). On the other hand, cell cycle analysis showed that treatment of HCT116 and SW480 cells with MRGBP siRNA significantly reduced cell population in S-phase compared with control siRNA $(35.4 \pm 2.0 \%$ vs $17.5 \pm 1.2 \%$ in HCT116, $P=0.0002 ; 29.4 \pm 1.3 \%$ vs $26.4 \pm 0.2 \%$ in SW480, $P=0.016$ ). Consistently, DNA synthesis was suppressed by MRGBP siRNA compared with control siRNA (Figure 2C). These results suggested that MRGBP might have an essential function in proliferation of colorectal cancer cells through regulation of cell cycle.

\section{Identification of bromodomain containing 8 as an MRGBP-interacting protein}

To further investigate the function of MRGBP, we performed yeast two-hybrid screening and identified BRD8 (also known as skeletal muscle abundant protein (SMAP) and p120) as an MRGBPinteracting protein (data not shown). As all 32 positive clones contained the C-terminal region of BRD8, the region was likely to be responsible for the interaction. To confirm the interaction between MRGBP and BRD8, immunoprecipitation assay was performed using plasmids expressing Myc/His-tagged MRGBP (pcDNA-Myc/His-MRGBP) and HA-tagged BRD8 (pCAGGS-HABRD8). When COS7 cells were transfected with both pcDNA-Myc/ His-MRGBP and pCAGGS-HA-BRD8, immunoprecipitation with anti-HA antibody followed by immunoblotting with anti-Myc antibody showed a single band corresponding to Myc-tagged MRGBP. Consistently, immunoprecipitation with anti-Myc antibody co-precipitated HA-tagged BRD8 (Figure 3A). We further examined endogenous interaction of MRGBP with BRD8 using nuclear extract from SW480 cells. As shown in Figure 3B (upper panels), immunoprecipitation with anti-MRGBP antibody coprecipitated endogenous BRD8. In addition, usage of anti-BRD8 antibody for immunoprecipitation also showed interacting endogenous MRGBP with BRD8 (Figure 3B, lower panels). BRD8 has been reported to express three transcript variants. Isoform 1 of 

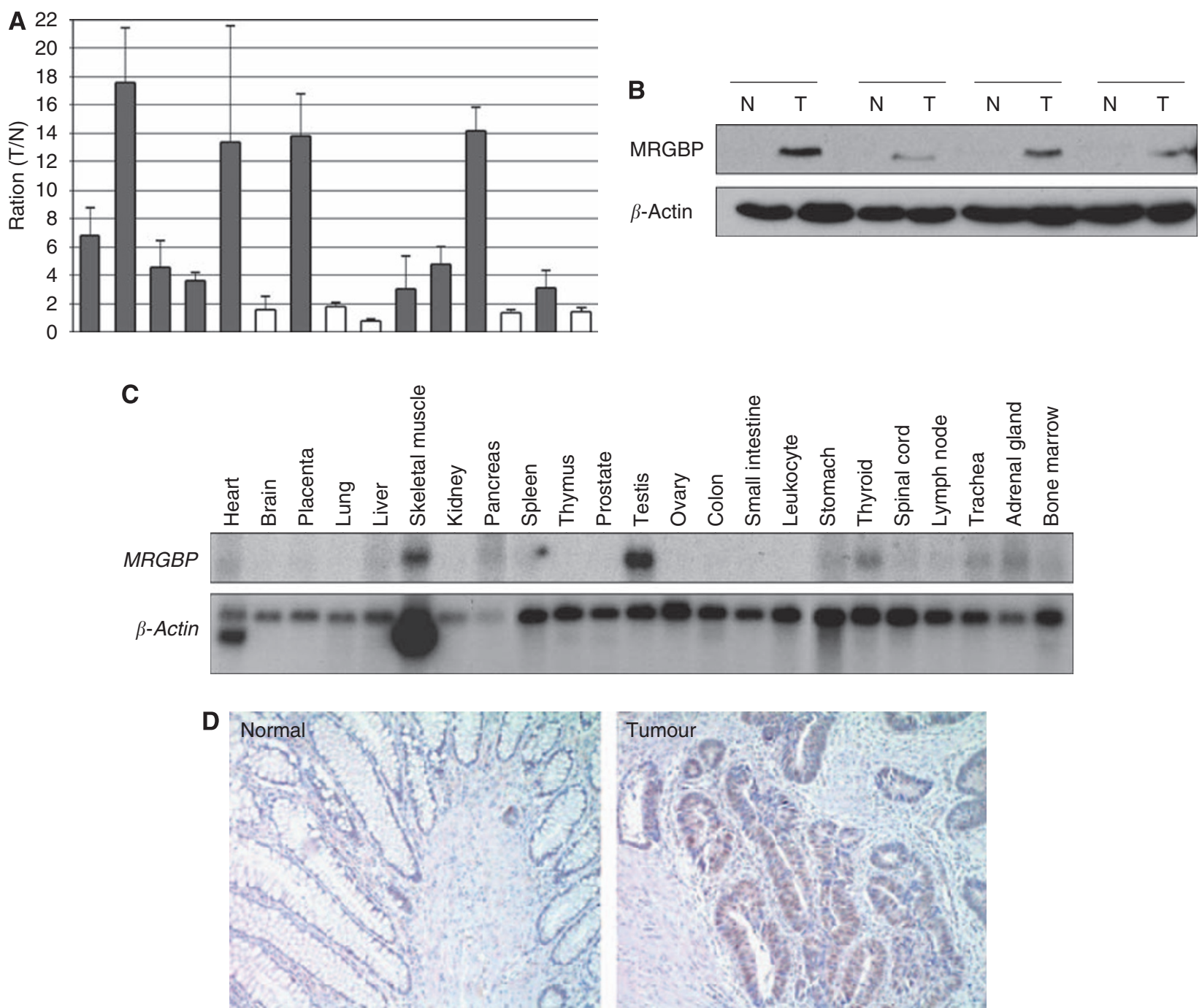

Figure I MRGBP is overexpressed in human colorectal tumours. (A) Relative expression of MRGBP in I5 additional colorectal tumours and the corresponding non-cancerous mucosa was analysed by real-time PCR. Quantity of MRGBP was normalised to HPRTI expression. The $y$ axis indicates the ratio of mean of MRGBP expression in tumour to that in normal tissues. The data represents mean \pm s.d. from three independent experiments. (B) Representative western blotting result of MRGBP in normal and tumour tissues from human colon. Expression of $\beta$-actin served as a control. (C) Multipletissue northern blot analysis of MRGBP in a panel of 23 normal human adult tissues. Expression of $\beta$-actin served as a control. (D) Representative images of immunohistochemical staining of MRGBP in normal and tumour tissues from human colon. Magnification: $\times 100$.

$B R D 8$, which we cloned, represents the predominant transcript. Isoform 2 encodes a protein with a longer and different C-terminal region compared with isoform 1. Although BRD8 isoform 1 contains one bromodomain, isoform 2 contains two. We additionally performed immunoprecipitation experiment using isoform 2 expression plasmid. As a result, isoform 2 also interacted with MRGBP (Supplementary Figure 1A).

\section{Responsible region of MRGBP for the interaction with BRD8}

To address the responsible region of MRGBP for the interaction with BRD8, we prepared various deletion mutants of MRGBP (Figure 3C). Immunoprecipitation and subsequent immunoblot analysis disclosed that wild type and MRGBP $\Delta 1$, an N-terminal deletion mutant containing codons 24-204 bound with BRD8. However, MRGBP $\triangle 2$ containing codons 44-204 did not associate with BRD8. In addition, MRGBP $\Delta 4$, another deletion mutant containing codons 1-90 interacted with BRD8, whereas MRGBP $\Delta 3$ containing codons 1-76 did not (Figure 3C). These data indicated that codons 24-90 could be essential for the interaction.

\section{MRGBP increases BRD8 protein in a post-transcriptional manner}

To examine the levels of BRD8 expression resulting from the interaction with MRGBP, we carried out western blot analysis. Compared with cells expressing exogenous BRD8 alone, the presence of MRGBP markedly enhanced expression of BRD8. The induced BRD8 expression was dependent on the time of transfection, with a continuous increase up to $48 \mathrm{~h}$. In contrast, the levels of BRD8 were unchanged without MRGBP (Figure 4A, upper panels). We also analysed $B R D 8$ mRNA in the cells by northern blot analysis. As shown in Figure 4A (lower panels), the levels of $B R D 8$ mRNA were not affected by MRGBP, indicating that the MRGBP-induced BRD8 protein results from post-transcriptional mechanisms. As another experiment showed that MG132, a proteasome inhibitor, greatly enhanced BRD8 protein (Supplementary 

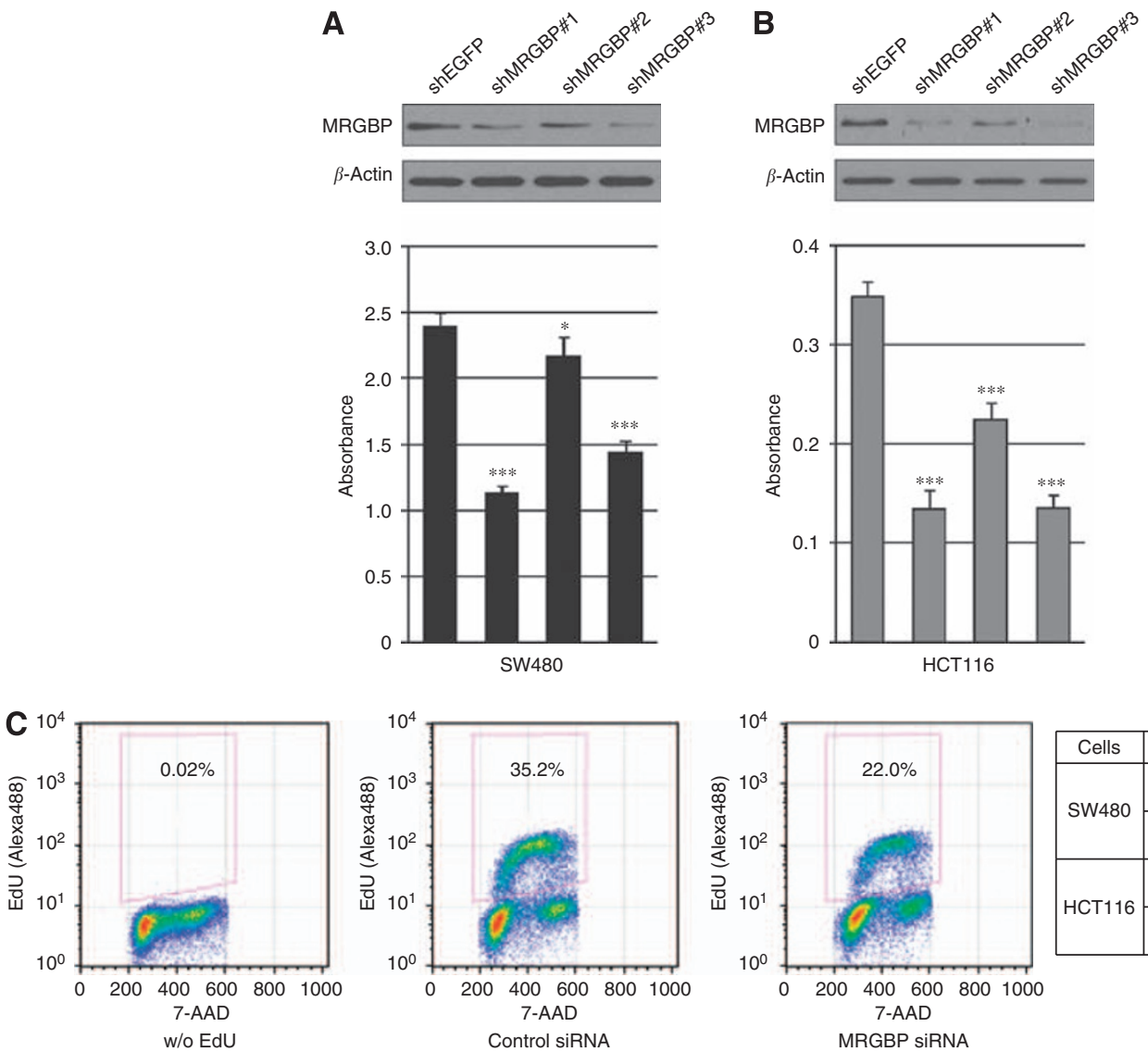

$\begin{array}{|c|c|c|}\hline \text { Cells } & \text { siRNA } & \text { EdU-positive (\%) } \\ \hline \multirow{2}{*}{\text { SW480 }} & \text { Control } & 37.3 \pm 0.3 \\$\cline { 2 - 3 } & \text {$\left.MRGBP } & 32.3 \pm 0.4\end{array}\right] P=0.0001$

Figure 2 Effect of MRGBP shRNA on the proliferation of colorectal cancer cells. (A) SW480 and (B) HCTI I 6 cells were treated with MRGBP shRNAs or EGFP shRNA (control) for $48 \mathrm{~h}$, and western blot analysis was performed. Expression of $\beta$-actin served as a control. Viability of cells transfected with shRNAs was measured by cell proliferation assay kit. The data represents mean \pm s.d. from five independent transfections. A significant difference was determined by Student's t-test; *, $P<0.05$; ***, $P<0.00$ I, vs EGFP shRNA-transfected cells. (C) SW480 and HCTI I 6 cells were treated with control or MRGBP siRNA for $48 \mathrm{~h}$, and then were incubated with $10 \mu \mathrm{M}$ EdU for $30 \mathrm{~min}$. Representative flow cytometric results of HCTI I 6 cells without EdU incorporation (left) and the cells transfected with control siRNA (middle) or MRGBP siRNA (right) were shown. The data represents mean \pm s.d. from three independent transfections. A significant difference was determined by Student's $t$-test.

Figure 1B), the degradation might have an important function in regulating BRD8 expression. Consistent with this view, wild type and mutant MRGBP ( $\Delta 1$ and $\Delta 4)$ that associated with BRD8 increased BRD8 expression (Figure 3C). However, mutant MRGBP $(\mathrm{MRGBP} \Delta 2$ and $\Delta 3$ ) lacking the binding ability with BRD8 did not enhance BRD8 expression (Figure 3C). To confirm the evidence that MRGBP regulates BRD8 expression, we finally knocked down endogenous MRGBP using siRNA. In complete agreement with the result of elevated BRD8 expression by MRGBP, knockdown of MRGBP substantially down-regulated BRD8 expression (Figure 4B). These results identified BRD8 as a novel down-stream target of MRGBP.

\section{DISCUSSION}

We have shown for the first time that MRGBP (C20orf20) is upregulated in the majority of colorectal cancer, and that its elevated expression is implicated in the proliferation of cancer cells. In addition, we have discovered that MRGBP associates with BRD8. Analysis of TRRAP/TIP60 complex by mass spectrometry identified a number of components including MRGBP (Cai et al, 2003). Consistent with our finding, BRD8 was also included in the complex (Cai et al, 2003). As all positive yeast clones contained bromodomain in the C-terminal region, bromodomain might be responsible for the binding. Three alternatively spliced forms of
BRD8 transcripts have been reported, and all forms include one or two bromodomains at their C-terminal. The predominant variant of transcripts encodes p120 (BRD8 isoform 1), a coactivating factor for thyroid hormone receptor (Monden et al, 1997). Interestingly, p120 was also found to interact with PPAR $\gamma / \mathrm{RXR}$ heterodimer on PPAR-response elements in the presence of the ligand (Monden et al, 1999), suggesting that p120 should be involved in transcriptional regulation. BRD8 isoform 2 contains two bromodomains and has a longer C-terminus compared with p120. Variant 3 encodes SMAP (or BRD8 isoform 3), which was isolated as a highly expressed transcript in skeletal muscle (Nielsen et al, 1996). However, the function of SMAP has not been clarified. In addition to isoform 1, we confirmed that MRGBP associates with isoform 2. As isoform 3 shares the same bromodomain with isoform 1, MRGBP should also interact with isoform 3.

In this study, we also examined co-localisation of MRGBP and BRD8 by immunocytochemical staining (Supplementary Figure 2). Consistent with the data of immunohistochemical analysis of MRGBP, exogenous MRGBP protein was accumulated in the nucleus of COS7 cells (Supplementary Figure 2A). On the other hand, BRD8 was mainly localised in the cytoplasm (Supplementary Figure 2B). Interestingly, when COS7 cells were transfected with both plasmids expressing BRD8 and MRGBP, BRD8 accumulated in the nucleus and co-localised with MRGBP (Supplementary Figure 2C). These data implicate that MRGBP alters subcellular 


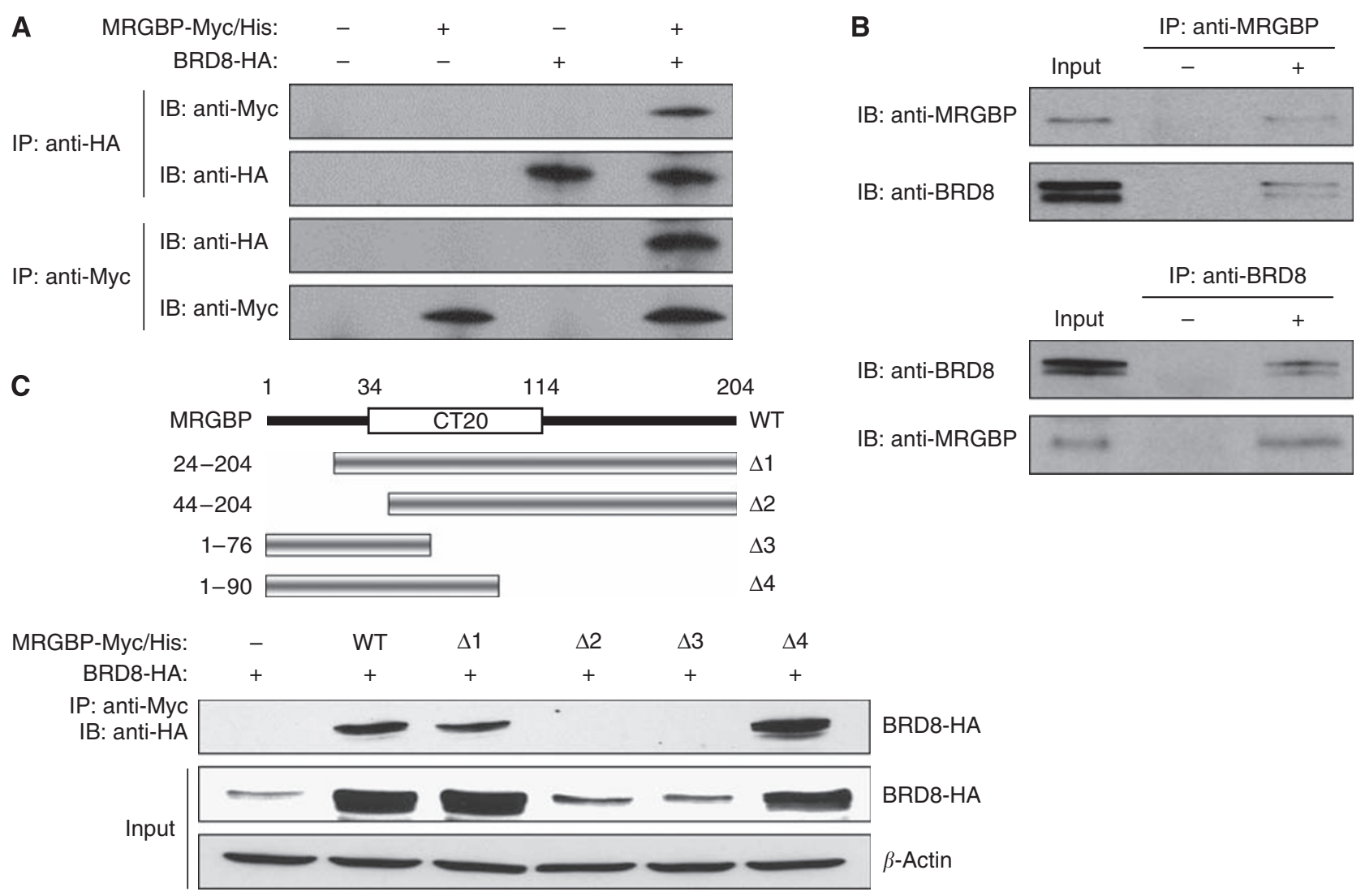

Figure 3 Interaction between MRGBP and BRD8. (A) COS7 cells were transfected with pcDNA-Myc/His-MRGBP, pCAGGS-HA-BRD8, or the combination of the two. Extracts from these cells were immunoprecipitated with anti-HA (upper two panels) or anti-Myc antibody (lower two panels). (B) Nuclear extract from SW480 cells were immunoprecipitated with anti-MRGBP (upper two panels) or anti-BRD8 antibody (lower two panels). Western blot analysis was performed using the indicated antibodies. (C) Interaction of wild type or the deletion mutants of MRGBP with BRD8. HEK293 cells were transfected with HA-tagged BRD8 plasmid and wild type or several deletion mutants of Myc-tagged MRGBP plasmids. Extracts from these cells were immunoprecipitated with anti-Myc antibody and then immunoblotted with anti-HA antibody (upper panel). Expressions of HA-tagged BRD8 and $\beta$-actin were shown in the middle panel and the lower panel, respectively.
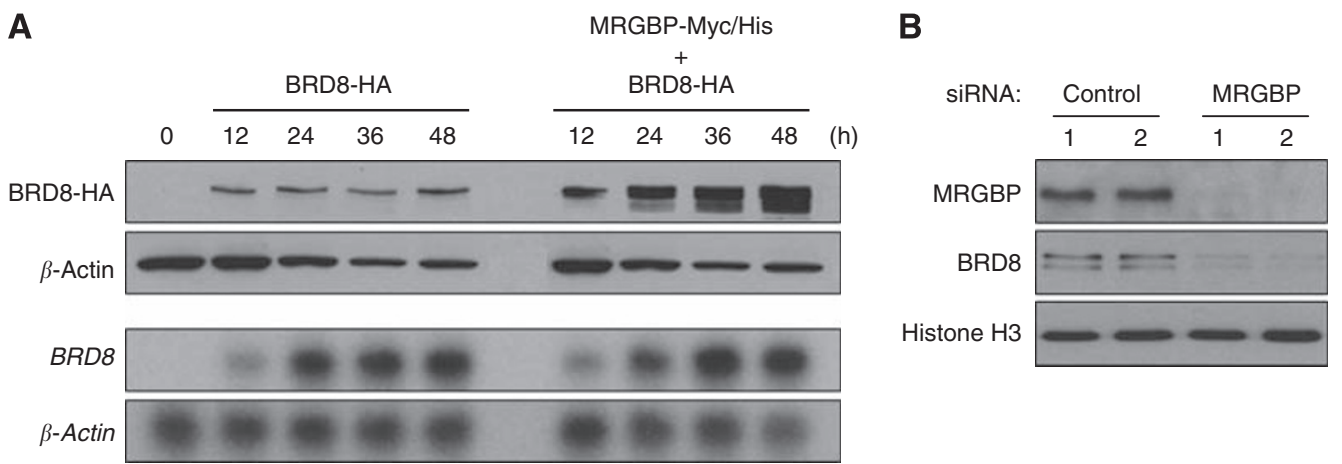

Figure 4 MRGBP increases expression of BRD8 protein in a post-transcriptional manner. (A) HEK293 cells were transfected with pcDNA-Myc/HisMRGBP and/or pCAGGS-HA-BRD8. After transfection, the cells were harvested at the indicated time points, and western blot analysis was performed (upper two panels). Expression of $\beta$-actin served as a control. Simultaneous northern blot analysis using BRD8 or $\beta$-actin cDNA as a probe was performed (lower two panels). Expression of $\beta$-actin served as a control. (B) Effect of knockdown of MRGBP on BRD8 expression. HCTI I 6 cells were transfected with MRGBP-specific or control siRNA for $72 \mathrm{~h}$. Nuclear extracts were isolated, and western blot analysis was performed using the indicated antibodies. Expression of Histone $\mathrm{H} 3$ served as a control.

localisation of BRD8 and that it increases BRD8 expression in a post-transcriptional manner. As proteasome inhibitor MG132 strikingly augmented BRD8 expression, BRD8 protein is likely to be easily degraded in the proteasome (Supplementary Figure 1B). Taken together, these data suggest that MRGBP may regulate the stability of BRD8. We also found that the interaction of MRGBP with BRD8 is essential for the MRGBP-induced BRD8 accumulation
(Figure 3C). Therefore, MRGBP may participate in the shuttling of BRD8 into the nucleus in which proteolysis machinery is inactive. Furthermore, to address the function of BRD8 on cell proliferation, we conducted cell proliferation experiment using $B R D 8$ shRNA construct. Treatment of HCT116 cells with BRD8 or control shRNA showed that proliferation of HCT116 cells was significantly reduced by BRD 8 shRNA compared with control shRNA (Supplementary 
Figure 2D). Therefore, BRD8 may have an important function for cell proliferation as a down-stream target of MRGBP in cancer cells. Although this hypothesis should be investigated in future studies, our findings have uncovered a novel function of MRGBP that is a member of the TRRAP/TIP60 complex.

In summary, the expression of MRGBP is enhanced in the majority of colorectal cancers, and its expression is associated with the growth of cancer cells. Interaction of MRGBP with BRD8 is probably a key for determination of MRGBP function in cancer cells. Our findings will be helpful for the profound understanding of colorectal carcinogenesis and may contribute to the development of novel anti-cancer drugs.

\section{REFERENCES}

Cai Y, Jin J, Tomomori-Sato C, Sato S, Sorokina I, Parmely TJ, Conaway RC, Conaway JW (2003) Identification of new subunits of the multiprotein mammalian TRRAP/TIP60-containing histone acetyltransferase complex. J Biol Chem 278: $42733-42736$

Fukuoka M, Yano S, Giaccone G, Tamura T, Nakagawa K, Douillard JY, Nishiwaki Y, Vansteenkiste J, Kudoh S, Rischin D, Eek R, Horai T, Noda K, Takata I, Smit E, Averbuch S, Macleod A, Feyereislova A, Dong RP, Baselga J (2003) Multi-institutional randomized phase II trial of gefitinib for previously treated patients with advanced non-small-cell lung cancer (The IDEAL 1 Trial) [corrected]. J Clin Oncol 21: 2237-2246

Giantonio BJ (2006) Bevacizumab in the treatment of metastatic colorectal cancer (mCRC) in second- and third-line settings. Semin Oncol 33: $\mathrm{S} 15-\mathrm{S} 18$

Gridelli C, Rossi A, Maione P (2003) Treatment of non-small-cell lung cancer: state of the art and development of new biologic agents. Oncogene 22: 6629-6638

Hurwitz H, Fehrenbacher L, Novotny W, Cartwright T, Hainsworth J, Heim W, Berlin J, Baron A, Griffing S, Holmgren E, Ferrara N, Fyfe G, Rogers B, Ross R, Kabbinavar F (2004) Bevacizumab plus irinotecan, fluorouracil, and leucovorin for metastatic colorectal cancer. $N$ Engl $J$ Med 350: 2335-2342

Kris MG, Natale RB, Herbst RS, Lynch Jr TJ, Prager D, Belani CP, Schiller JH, Kelly K, Spiridonidis H, Sandler A, Albain KS, Cella D, Wolf MK, Averbuch SD, Ochs JJ, Kay AC (2003) Efficacy of gefitinib, an inhibitor of the epidermal growth factor receptor tyrosine kinase, in symptomatic patients with non-small cell lung cancer: a randomized trial. JAMA 290: 2149-2158

Lin YM, Furukawa Y, Tsunoda T, Yue CT, Yang KC, Nakamura Y (2002) Molecular diagnosis of colorectal tumors by expression profiles of 50 genes expressed differentially in adenomas and carcinomas. Oncogene 21: $4120-4128$

\section{ACKNOWLEDGEMENTS}

We thank Yoshika Sakamoto for her technical assistance, Yumiko Ishii (IMSUT FACS Core Laboratory) for the assistance of flow cytometry, and Drs Masaki Kunizaki and Fabio Pittella Silva for helpful discussions. This work was supported in part by Grant-inAid (\#17015009) for Scientific Research from The Ministry of Education, Culture, Sports, Science and Technology Japan.

Supplementary Information accompanies the paper on British Journal of Cancer website (http://www.nature.com/bjc)
Molina MA, Codony-Servat J, Albanell J, Rojo F, Arribas J, Baselga J (2001) Trastuzumab (herceptin), a humanized anti-Her2 receptor monoclonal antibody, inhibits basal and activated Her2 ectodomain cleavage in breast cancer cells. Cancer Res 61: 4744-4749

Monden T, Kishi M, Hosoya T, Satoh T, Wondisford FE, Hollenberg AN, Yamada M, Mori M (1999) p120 acts as a specific coactivator for 9-cisretinoic acid receptor (RXR) on peroxisome proliferator-activated receptor-gamma/RXR heterodimers. Mol Endocrinol 13: 1695-1703

Monden T, Wondisford FE, Hollenberg AN (1997) Isolation and characterization of a novel ligand-dependent thyroid hormone receptor-coactivating protein. J Biol Chem 272: 29834-29841

Nielsen MS, Petersen CM, Gliemann J, Madsen P (1996) Cloning and sequencing of a human cDNA encoding a putative transcription factor containing a bromodomain. Biochim Biophys Acta 1306: 14-16

O'Dwyer ME, Druker BJ (2000) Status of bcr-abl tyrosine kinase inhibitors in chronic myelogenous leukemia. Curr Opin Oncol 12: 594-597

Parkin DM (2001) Global cancer statistics in the year 2000. Lancet Oncol 2: $533-543$

Shimokawa T, Furukawa Y, Sakai M, Li M, Miwa N, Lin YM, Nakamura Y (2003) Involvement of the FGF18 gene in colorectal carcinogenesis, as a novel downstream target of the beta-catenin/T-cell factor complex. Cancer Res 63: 6116-6120

Tabernero J, Van Cutsem E, Diaz-Rubio E, Cervantes A, Humblet Y, Andre T, Van Laethem JL, Soulie P, Casado E, Verslype C, Valera JS, Tortora G, Ciardiello F, Kisker O, de Gramont A (2007) Phase II trial of cetuximab in combination with fluorouracil, leucovorin, and oxaliplatin in the first-line treatment of metastatic colorectal cancer. J Clin Oncol 25: $5225-5232$

Wakeling AE (2002) Epidermal growth factor receptor tyrosine kinase inhibitors. Curr Opin Pharmacol 2: 382-387 